\title{
MS1 Quantification by Isotope Labeling
}

National Cancer Institute

\section{Source}

National Cancer Institute. MS1 Quantification by Isotope Labeling. NCI Thesaurus. Code C161815.

A proteomic quantitation method where isotopically-labeled peptides are quantified during a single round of mass spectrometry. 